\title{
Ear asymmetry and the temporal uncertainty of signals in sustained attention
}

\author{
JOEL S. WARM, DONALD A. SCHUMSKY, and DOUGLAS K. HAWLEY \\ University of Cincinnati, Cincinnati, Ohio 45221
}

\begin{abstract}
Subjects listened for increments in the duration of recurrent white noise pulses delivered to either the left or the right ear during a 1-h vigilance session. The temporal uncertainty of critical signal occurrences was varied by use of signal densities of 24,48 , and 96 signals/hour. Response times to signal detections increased as a function of an information measure of the temporal uncertainty of signals with a markedly stronger effect from left-ear presentation. Thus, a functional lateral asymmetry in the cortical processing of temporal information in this type of task was indicated.
\end{abstract}

Temporal uncertainty or uncertainty as to when a critical signal will appear is an important feature of most sustained attention or vigilance tasks. One means of studying this feature experimentally is through variations in signal density or the rate of appearance of critical signals. The more frequently such signals occur, the less the subject's average uncertainty about when they will occur, and typically, the greater the efficiency of his performance (Loeb \& Alluisi, 1970).

A series of studies by Smith, Warm, and Alluisi (1966) and Warm and Alluisi (1971) has introduced an information theory analysis into the temporal uncertainty problem. These investigators derived an information or uncertainty metric to provide a measure of the density-determined temporal uncertainty regarding when a critical signal will occur. They reported that over a range of 6 to 96 signals/hour, the subject's response time (RT) could be represented as a monotonically increasing function of signal uncertainty due to density. Furthermore, this effect was equally observable with both acoustic and visual signals. Such findings led to the conclusion that some centrally located mechanism for the processing of temporal information plays an important role in vigilance performance.

In recent years, it has become evident that the information handling capabilities of human observers in a variety of perceptual situations is based, in part. upon differential specialization in the functional properties of the cortical hemispheres. For example, it is generally accepted that the left hemisphere is specialized for processing verbal information while the right hemisphere may be dominant for several other nonverbal functions involving spatial infor-

Supported in part by the Institute of Space Sciences of the University of Cincinnati under National Aeronatics and Space Administration Grant NGL-36-004-014. Requests for reprints should be sent to Joel S. Warm, Department of Psychology, University of Cincinnati, Cincinnati, Ohio 45221. mation (Bever, 1975). To date, few attempts have been made to determine if hemispheric differences may be involved in the processing of temporal information in general, and in sustained attention tasks in particular. Consequently, the purpose of this experiment was to explore the possibility that the cerebral hemispheres may be differentially sensitive to the effects of temporal uncertainty in the vigilance task.

Attempts to isolate disparities in hemispheric functions require the differential presentation of stimuli to each hemisphere. The most obvious means of accomplishing this would be to utilize a divided-field technique in order to deliver stimuli to the right or left hemi-retina. Such a procedure is difficult in the vigilance situation since subjects are typically tested for long periods of time and without restraint. Therefore, the proper orchestration of eye movements and the retinal locus of stimulation cannot be easily accomplished. Several experiments on hemispheric asymmetry have made use of the fact that like the visual system, the auditory system is also crossed and contralateral connections between the ear and the cortex are stronger and more effective than ipsilateral connections (Doehring, 1972; Murphy \& Venables, 1970; Robinson \& Solomon, 1974; Rosenzweig, 1951). Thus, asymmetries in performance associated with ear of presentation have been considered to reflect asymmetries in hemispheric specialization. For these reasons, acoustic stimulation was used in this investigation. The strategy was to probe for hemispheric differences by examining the effects of the temporal uncertainty of signals delivered to the left or to the right ear.

\section{METHOD}

Forty-eight undergraduates, 30 men and 18 women, served as subjects. They ranged in age from 17 to 31 , with a mean of 20 years. All subjects were free of any known hearing impairment and were judged to be right dominant in tests of usage of eye, hand, and foot. None 
of the students had previous experience in vigilance or hemispheric dominance experiments.

All subjects participated in a 1-h session during which they listened for occasional increments in the duration of recurrent white noise pulses. Throughout the session, $.5-\mathrm{sec}$ pulses of stimulation were presented once every $2.0 \mathrm{sec}$. These constituted nonsignal events to which no overt response was required. A $1.0-\mathrm{sec}$ pulse (twice the normal duration) was the critical signal for detection. Subjects responded to critical signals by depressing a push-button switch with their right hand. The response switch was mounted on a flat-black panel and located on a table approximately $71 \mathrm{~cm}$ from the seated subject. In order to reduce variability due to motor movement time, the subjects were instructed to keep the index finger of the right hand on a small white dot immediately below the response switch. Acoustic stimulation was delivered through a pair of headphones at an intensity of $78 \mathrm{~dB}$ SPL. Appropriate switching circuitry permitted the presentation of the white noise pulses either to the left or to the right ear, separately.

Three levels of critical signal density, 24, 48, and 96 signals/hour were employed. Sixteen subjects were assigned at random to each density level with the restriction that 10 men and 6 women appear in each condition. The average intersignal intervals (ISIs) for these density levels were 150,75 , and $36.5 \mathrm{sec}$, respectively. Within each density condition, the statistical distribution of ISIs approximated one half of a normal distribution with a unit coefficient of variation; the sequence of ISIs was randomly ordered for each subject. The ISI distribution and the signal densities were identical to those used by Smith, Warm, and Alluisi (1966) and Warm and Alluisi (1971).

As described in these earlier reports, the densitydetermined temporal uncertainty of signals may be expressed as:

$$
h=-\log _{2} P(\text { signal }),
$$

where $h$ is the information, in bits, associated with the signal's occurrence at a specific time, and the probability of occurrence of a signal in any specified interval is

$$
P(\text { signal })=D / D(\max ),
$$

in which $D$ is the signal density or actual number of signals per hour and $\mathrm{D}(\max )$ is the maximum possible number of signals per hour. In the present experiment, pulses of stimulation were presented every $2.0 \mathrm{sec}$. Since there are $3,600 \mathrm{sec} /$ hour, $\mathrm{D}(\max )=1,800$. With signal densities (Ds) of 24, 48, and 96 signals/hour, the associated probabilities (Ps) are 24/1,800, 48/1,800, and $96 / 1,800$, and the uncertainties (hs) due to signal density are $6.23,5.23$, and 4.23 bits, respectively.

For each subject at all levels of signal density, acoustic pulses were presented to each ear alone for alternating 5 -min periods. The sequence in which the subjects experienced leftear rightear alternation was counterbalanced. At each density level, half of the total number of critical signals appeared during the first $1 / 2 \mathrm{~h}$ of the session and the remainder during the second $1 / 2 \mathrm{~h}$. Within each $1 / 2-\mathrm{h}$ period, equal numbers of critical signals appeared in the left and right ears.

The occurrence of neutral pulses and critical signals was controlled by solid state programming equipment and a punched tape timer. Response times to correct detections were read to the nearest millisecond from a digital electronic timer which was activated automatically $.5 \mathrm{sec}$ after the onset of a pulse comprising

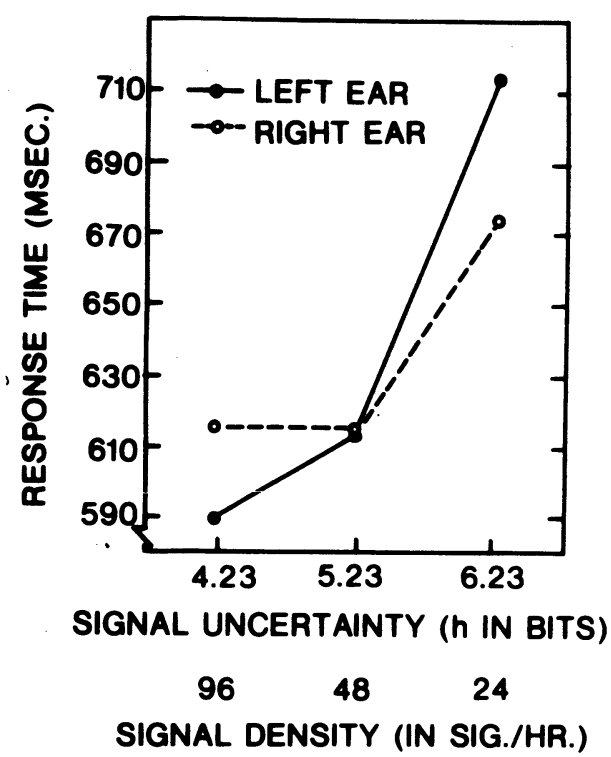

Figure 1. Ear asymmetry and the temporal uncertainty of signals. Response times to correct detections for left- and right-ear signals as a function of signal uncertainty. Signal density values are given for reference.

a critical event and terminated by the subject's response, or after a no-response duration of $6.0 \mathrm{sec}$. Failure to respond to a critical signal within $6 \mathrm{sec}$ after its termination was considered an error of omission (missed signal). Each subject was tested individually in a soundshielded room; the control equipment was located in an adjacent room. Subjects surrendered their watches at the start of the session and they had no knowledge as to the length of the vigil other than it would not exceed $2 \mathrm{~h}$.

\section{RESULTS}

Median RTs to correct detections were computed from the data of each subject for signals appearing in the left and right ears during the two $1 / 2$-h periods of the session. An analysis of variance of the data revealed that $\mathrm{RT}$ s increased significantly as time on watch progressed, the mean RTs for the first and second $1 / 2-h$ periods were 598 and $675 \mathrm{msec}$, respectively, $F(1,45)=33.13, p<.001$. The ear of presentation did not have a significant overall effect upon speed of signal detection, mean RTs for the left and right ears were 634 and $639 \mathrm{msec}$, respectively, $\mathrm{F}<1$. Variations in temporal uncertainty due to density also failed to exert a significant overall effect upon performance efficiency, mean RTs for signal densities of 24,48 , and 96 signals/hour were 694,613 , and $602 \mathrm{msec}$, respectively, $\mathrm{F}(2,45)=1.87, \mathrm{p}>.05$. However, there was a significant Ear by Uncertainty interaction, $F(2,45)=3.24, p<.05$. All of the remaining interactions lacked significance $(p>.05)$.

Response times for left and right ear signals are plotted as a function of signal uncertainty in Figure 1 (the corresponding signal densities are given for reference). 
It is evident in the figure, that the effects of signal uncertainty were relatively weak when probed by signals presented to the right ear. Response times for right ear signals were almost identical at the two lowest levels of uncertainty and increased by only $59 \mathrm{msec}$ at the highest level of temporal uncertainty. By contrast, with left ear signals, RTs increased consistently with increments in signal uncertainty and the difference in RT between the highest and lowest levels of uncertainty was $124 \mathrm{msec}$; a difference more than twice as large as that observed with right ear signals. ${ }^{1}$

An important methodological feature of this experiment was the shift in ear of stimulus presentation which occurred every $5 \mathrm{~min}$. In order to evaluate the effects of such shifts upon performance, the RT to the first postshift critical signal was examined against the RT to the last critical signal in each 5-min time block. These data were examined as a function of signal density, ear, and $1 / 2-h$ period of watch. Perusal of the data indicated that the magnitudes of the differences between mean RTs were far less than the associated standard deviations so that no further analysis seemed necessary. Indeed, the single largest difference between means was far less than the smallest cell standard deviation. We therefore conclude that the effects of the shift of ears per se did not make any substantial contribution to performance.

\section{DISCUSSION}

The results of this study are consistent with the previous finding that $\mathrm{RT}$ in the vigilance task increases as a function of the density-determined temporal uncertainty of signals (Smith, Warm, \& Alluisi, 1966; Warm \& Alluisi, 1971). In addition, the present results point to the possibility that the two cerebral hemispheres may function asymmetrically in processing information with regard to temporal uncertainty since increments in RT as a function of uncertainty were observable primarily when probed by signals appearing in the left ear.

The task used in this study was the same as that employed in the acoustic portion of the experiment by Warm \& Alluisi (1971) with the exception that acoustic stimulation was presented binaurally in the earlier report. Consequently, a comparison of the increments in RT noted in both experiments within a comparable range of signal uncertainties, 6.23 to 8.23 bits or signal densities of 96 to 24 signals/hour is instructive. In the earlier report, RTs to binaural acoustic signals increased by $80 \mathrm{msec}$ over this range; a value which lies approximately midway between the increment of $59 \mathrm{msec}$ found for right-ear signals in the present study and the increment of $124 \mathrm{msec}$ found for leftear signals. Taken together, the results from both experiments suggest that the two cerebral hemispheres may be jointly involved in organizing behavior in the vigilance task under conditions of temporal uncertainty. While the right hemisphere may be more sensitive to variations in uncertainty than the left, performance appears to reflect an averaging of the processing in both hemispheres. Such an arrangement could permit the organism to take advantage of conditions in which temporal uncertainty is relatively low without sacrificing performance efficiency too greatly when the conditions of uncertainty are relatively high.

Recently, Diamond and Beaumont (1973) have devised a clever means of coordinating the locus of signal presentations with eye movements in a visual vigilance task. They reported that the left hemisphere may be dominant in organizing behavior in this situation since detection probability was superior for signals presented to the left as compared to the right hemisphere. The results with acoustic signals and a latency measure in the present study are not totally consistent with those obtained by Diamond and Beaumont. Reexamination of Figure 1 reveals that while performance efficiency was superior for rightear (left hemisphere) relative to left-ear (right hemisphere) presentations at the highest level of signal uncertainty, just the opposite result occurred at the lowest level of signal uncertainty.

Comparisons of Diamond and Beaumont's results with those of the present study must be made with caution since the methodologies employed in the two experiments differ considerably. In addition to differences in mode of signal presentation (vision vs. audition) and response indices (detection probability vs. RT), Diamond and Beaumont probed for hemispheric effects at only one level of signal density (48 signals/ $100 \mathrm{~min}$ ) and their subjects were required to detect changes in signal intensity while duration increments were the critical events to be detected in the present study. The influence of these methodological factors will have to be explored in future research before the disparities between the two investigations can be reconciled.

One of the most ubiquitous features of performance in sustained attention tasks is the decrement function, the tendency for performance efficiency to decline over time. This effect was also noted in the present data; RTs to correct detections were longer in the second, relative to the first, $1 / 2$-h period of the session. Unlike the effects of signal uncertainty, however, the influence of time on task was not related to the ear of signal presentation and therefore to possible asymmetries in hemispheric functioning. This pattern of results may have important implications for theories of vigilance such as the expectancy and the arousal positions (Davies \& Tune, 1969) which attempt to incorporate the effects of both the temporal uncertainty of signals and time on task within the same explanatory framework. The fact that ear asymmetry was noted with respect to one parameter and not the other suggests that the effects associated with temporal uncertainty and time on watch may be mediated by different rather than by common processes.

\section{REFERENCES}

Bever. T. G. Cerebral asymmetries in humans are due to the differentiation of two incompatible processes: Holistic and analytic. Annals of the New York Academy of Sciences, 1975, 263, 251-262.

DAviEs, D. R., \& TUNE, G. S. Human vigilance performance. New York: American Elsevier, 1969.

Diamond, S. J., \& Beaumont, J. G. Difference in the vigilance performance of the right and left hemispheres. Cortex, $1973,9,259-265$.

Doenring, D. G. Ear asymmetry in the discrimination of monaural tone sequences. Canadian Journal of Psychology, 1972, 26. $106-110$

Loeb, M., \& Alluisi, E. A. Influence of display, task, and organismic variables on indices of monitoring behavior. Acta Psychologica, 1970, 33, 343-366. 
Murphy, E. H., \& Venables, P. H. The investigation of ear asymmetry by simple and disjunctive reaction-time tasks. Perception \& Psychophysics, 1970, 8, 104-106.

Robinson, G. M., \& Solomon, D. J. Rhythm is processed by the speech hemisphere. Journal of Experimental Psychology, 1974, 102. 508-511.

Rosenzweig, M. R. Representation of the two ears at the auditory cortex. American Journal of Physiology, 1951, 167, 147-158.

Smith, R. P., Warm, J. S., \& Alluisi, E. A. Effects of temporal uncertainty on watchkeeping performance. Perception \& Psychophysics, 1966, 1, 293-299.

WARM. J. S., \& Alluisi, E. A. Influence of temporal uncertainty and sensory modality of signals on watchkeeping performance. Journal of Experimental Psychology, 1971, 87, 303-308.

W ARM, J. S., EPps, B. D., \& Ferguson, R. P. Effects of knowledge of results and signal regularity on vigilance performance. Bulletin of the Psychonomic Society, 1974, 4, 272-274.

\section{NOTE}

1. Previous research with this task using acoustic signals has resulted in a very high level of detection (Warm \& Alluisi, 1971; Warm, Epps, \& Ferguson, 1974). This experiment was no exception; mean detection probabilities under all conditions ranged from .79 to .91 . No significant relationship was noted between levels of signal density or ear of presentation with regard to the rate of signal detections.

(Received for publication January 8, 1976.) 